\title{
ESTUDOS PRELIMINARES PARA A AVALIAÇÃO DA CAPACIDADE DE SOBREVIVÊNCIA DO ÁCARO Lynxacarus radovskyi (TENORIO, 1974) FORA DO HOSPEDEIRO
}

\author{
Edenilze Teles Romeiro, ${ }^{1}$ Leucio Câmara Alves ${ }^{2}$ e Maria Aparecida Gloria Faustino ${ }^{3}$ \\ 1.Professor adjunto I, Departamento de Medicina Veterinária da Universidade Federal Rural de Pernambuco. \\ E-mail: romeiroedenilze@click21.com.br \\ 2. Professor adjunto da Universidade Federal Rural de Pernambuco \\ 3. Professor adjunto IV da Universidade Federal Rural de Pernambuco.
}

\section{RESUMO}

Desenvolveu-se este estudo com o objetivo de avaliar a possibilidade de sobrevivência dos estágios do ciclo biológico do ácaro Lynxacarus radovskyi fora do hospedeiro em condições de laboratório. Coletaram-se ovos, formas imaturas e adultas do L. radovskyi, de felinos naturalmente parasitados. Os espécimes coletados foram separados e colocados em recipientes plásticos e em tubos de Durhan, sendo submetidos à temperatura e umidade ambiente, no Laboratório de Doenças Parasitárias dos Animais Domésticos - DMV - UFRPE, e em câmara climatizada para BOD com temperatura de $27 \pm 1^{\circ} \mathrm{C}$ e umidade relativa $\geq 70 \%$, em escotofase, com e sem fornecimento de pelo felino como substrato, para ser avaliada a capacidade de sobrevivência dos estágios fora do hospedeiro. Dos ovos coletados, houve eclosão de $100 \%$, com sobrevivência larval de um a quatro dias; para as larvas coletadas, a sobrevivência foi de um a cinco dias. Para todas as fases do experimento as ninfas apresentaram média de sobrevivência maior que os demais estágios. Em geral, as fêmeas sobreviveram mais que os machos. $\mathrm{O}$ aporte do pelo proporcionou uma sobrevivência maior para todos os estágios do ácaro.

PALAVRAS-CHAVES: Astigmata, estágios, gato, longevidade.

This study aimed to evaluate the survival possibility of the Lynxacarus radovskyi life cycle stages out of the host under laboratory conditions. Eggs, immature and adult forms of $L$. radovskyi were collected from cats naturally parasitized. The collected specimens were separated and put into plastic containers and into Durhan pipes, being submitted to room temperature and humidity, in the Parasitic Diseases of Domestic Animals Laboratory - DMV - UFRPE, and in climatic chamber at temperature of $27^{\circ} \mathrm{C} \pm 1{ }^{\circ} \mathrm{C}$ and relative humidity $\geq 70 \%$, in scotophase, with and without cat hair supply as substrate to evaluate the survival capacity of the stages out of the host. There was a $100 \%$ hatch of the collected eggs, with larval survival within one to four days; the survival for the collected larvae was from one to five days. For all the experiment phases the nymphs presented bigger survival average than the other ones. In general, females survived more than males. The addition of hair provided a bigger survival to all the mite stages.

KEYWORDS: Astigmata, cats, longevity, stages. 


\section{INTRODUÇÃO}

Como grande parte dos ácaros passa a vida em contato íntimo com seus hospedeiros, sua propagação é realizada primariamente por contato direto. $\mathrm{O}$ ciclo biológico básico possui quatro estágios evolutivos: ovo, larva hexápoda, ninfa octópoda e adulto. Em muitos casos, há vários ínstares ninfais antes do estágio adulto (GUIMARÃES et al., 2001).

Os ácaros da família Listrophoridae (CANESTRINI, 1892) são espécies de corpo delicado, fortemente estriado e com escudo dorsal distinto. Apresentam aparelho bucal e pernas adaptadas para segurarem-se nos pelos (GUIMARÃES et al., 2001), parasitando roedores, morcegos, carnívoros, primatas e marsupiais (FLECHTAMNN, 1975).

Com relação ao gênero Lynxacarus, existem sete espécies descritas: Lynxacarus mustelac Megnin, 1885 em Carnivora: Mustelidae; L. morlani Radforf, 1951 em Carnivora: Felidae; L. dubinini, 1969 em Insectivora: Talpidae; L. lyncodon Fain, $1970 \mathrm{em}$ Carnivora: Mustelidae; L. semnopitheci Fain, 1970 em Primata: Cercopithecidae; L. tupaiae Fain, 1970 em Primata: Tupaiidae; L. nearcticus Fain e Hyland, 1973 em Carnivora: Mustelidae. No gato doméstico, Lynxacarus radovskyi foi reconhecido e relatado como ácaro pilícola parasito, evidenciando-se, também, a sua importância na clínica de felinos, sendo a segunda espécie de ácaro que mais acometia os felinos domésticos nas Ilhas do Hawaii (TENORIO, 1974).

O período de desenvolvimento dos ácaros (ovo até adulto) varia de acordo com a alimentação, temperatura e umidade do ambiente em que eles se encontram. Na dependência desses fatores, o período entre uma e outra fase evolutiva pode variar grandemente, sobretudo, com as variações microclimáticas. Em média, o ciclo evolutivo transcorre num prazo de vinte a trinta dias em temperatura entre $18{ }^{\circ} \mathrm{C}$ e $30^{\circ} \mathrm{C}$ e umidade relativa do ar entre $60 \%$ e $70 \%$ (CROCE \& BAGGIO, 1993).

Em virtude do achado de inúmeros indivíduos adultos (machos e fêmeas), ninfas, visualização do ovo no interior de muitas fêmeas, como também da presença de ovos nos pelos de felinos domésticos naturalmente parasitados, segundo diversos autores (TENORIO, 1974; FOLEY, 1991; CRAIG et al., 1993; PEREIRA, 1996), o ciclo completo do L. radovskyi, provavelmente, ocorre sobre o animal. Portanto, objetivou-se neste experimento avaliar a possibilidade de sobrevivência dos estágios do ciclo biológico do ácaro Lynxacarus radovskyi fora do hospedeiro em condições de laboratório.

\section{MATERIAL E MÉTODOS}

$\mathrm{O}$ experimento realizou-se em três fases. Em cada uma delas, o número de espécimes utilizados variou de acordo com a disponibilidade de material coletado. Realizou-se a coleta por meio da avulsão de pelos (SLOSS et al., 1999) em animais da espécie felina, de ambos os sexos, raça e idade variadas procedentes do Gatil do Departamento de Medicina Veterinária (DMV), Universidade Federal Rural de Pernambuco (UFRPE), naturalmente parasitados. Os pelos foram acondicionados em recipientes plásticos com tampa, tipo coletor universal, devidamente fechados e mantidos em temperatura ambiente até a chegada ao Laboratório de Doenças Parasitárias dos Animais Domésticos (DMV - UFRPE), para processamento do material. Imediatamente após a coleta, cada amostra de pelo com ácaros foi colocada em placa de Petri e observada em microscópio estereoscópico, para determinação do estágio do ciclo de cada espécime, conforme características morfológicas descritas por TENORIO (1974) e FACCINI \& COUTINHO (1986).

A temperatura e a umidade ambiente foram aferidas diariamente, mediante um termo-higrômetro digital modelo TERMOHYGRO - TFA, sendo anotadas em ficha preestabelecida.

Na primeira fase (Fase 1), utilizaram-se recipientes plásticos transparentes de formato redondo, com aproximadamente $3 \mathrm{~cm}$ de diâmetro e $2 \mathrm{~cm}$ de altura, com tampa rosqueada de fechamento externo, perfurada previamente no centro com um furo único de aproximadamente $1,5 \mathrm{~cm}$ de diâmetro. Esse orifício foi recoberto com uma tela de nylon de 200 micras, fixada com cola quente de silicone. Realizaram-se as coletas de maio a agosto de 2005 , totalizando 23 ovos, cinco larvas, 46 ninfas, 58 machos, 83 fêmeas, sendo introduzido um espécime do ácaro em cada recipiente, além de um casal mantido em um recipiente.

Os recipientes foram mantidos sobre bancada em temperatura e umidade ambiente em recinto 
fechado com luminosidade natural, no Laboratório de Doenças Parasitárias dos Animais Domésticos, sendo observados diariamente, até a morte do ácaro. Desenvolveram-se as observações a cada 24 horas em microscópio estereoscópico, apenas neste momento submetidos à luz artificial.

Na segunda fase (Fase 2), procedeu-se às coletas em novembro e dezembro de 2005. Utilizaram-se tubos de Durhan, vedados com algodão hidrófilo, mantendo-se uma parte em temperatura ambiente no mesmo recinto anteriormente mencionado, sobre bancada, sendo os tubos posicionados verticalmente, apoiados em placa de micropoços de poliestireno, e outra parte mantida em câmara climatizada para BOD com temperatura de $27 \pm 1^{\circ} \mathrm{C}$ e umidade relativa $\geq 70 \%$, em escotofase. As condições de luminosidade para os tubos mantidos em temperatura ambiente foram iguais às da primeira fase do estudo. Colocaram-se em temperatura ambiente 31 larvas, 71 ninfas, 63 machos e 98 fêmeas, e em BOD 8 ovos, 25 larvas, 80 ninfas, 68 machos, 131 fêmeas e cinco casais. Os tubos foram observados diariamente, em microscópio estereoscópico, como descrito na primeira fase.

$\mathrm{Na}$ terceira fase (Fase 3) do experimento, realizaram-se as coletas de dezembro de 2005 a janeiro de 2006. Seguiu-se procedimento semelhante ao da segunda fase, porém os tubos foram mantidos em temperatura ambiente e acrescentaram-se a estes pelos de felinos como fonte de alimentação, sendo colocados oito ovos, 53 larvas, 139 machos, 150 fêmeas e 94 ninfas. As observações ocorreram diariamente como nas fases anteriores.

Para análise estatística, empregaram-se técnicas do tipo descritiva, por meio de distribuições absolutas e percentuais do número de espécimes, utilizando-se média e desvio padrão. Foram utilizadas também técnicas de estatística inferencial por meio dos testes t-Student com variâncias iguais ou desiguais, F (ANOVA), com comparações de Tukey. Efetuou-se a verificação da hipótese de igualdade de variâncias através do teste $\mathrm{F}$ de Levene (ALTMAN, 1991; ZAR, 1999). Para a obtenção dos cálculos estatísticos, utilizou-se o programa Statistical Package for the Social Sciences (SPSS) na versão 13, com margem de erro de 5,0\%, empregada para a decisão dos testes estatísticos.

\section{RESULTADOS E DISCUSSÃO}

$\mathrm{Na}$ primeira fase do experimento a temperatura e a umidade ambiente variaram de $25,8{ }^{\circ} \mathrm{C}$ a 30 ${ }^{\circ} \mathrm{C}$ e $62 \%$ a $84 \%$, com médias de $26,8{ }^{\circ} \mathrm{C}$ e $71,27 \%$, respectivamente; na segunda fase, a variação foi de $28,5{ }^{\circ} \mathrm{C}$ a $31^{\circ} \mathrm{C}$ e $50 \%$ a $70 \%$, com médias de $27,4{ }^{\circ} \mathrm{C}$ e $58 \%$ respectivamente; e, na terceira fase, a temperatura ambiente e a umidade relativa apresentaram, respectivamente, variações de $28{ }^{\circ} \mathrm{C}$ a $31^{\circ} \mathrm{C}$ e $52 \%$ a $70 \%$, com médias de $30{ }^{\circ} \mathrm{C}$ e $60,8 \%$.

Avaliando-se os dados obtidos na primeira fase, dos ovos colocados $100 \%$ deram origem a larvas que sobreviveram um dia. Observou-se que algumas larvas apresentaram dificuldade para sair da casca ovígera, encontrando-se 39,1\% (9/23) presas parcialmente à casca (Figura 1), sendo retiradas com $\mathrm{o}$ auxílio de um pincel adaptado de $\mathrm{n}^{\mathrm{o}} 0$.

As larvas coletadas sobreviveram de um a dois dias, sendo que $80 \%$ morreram com dois dias; as ninfas sobreviveram de três a dez dias, com um percentual maior $(32,6 \%)$ morrendo com cinco dias. Os machos apresentaram sobrevivência de um a quatro dias e as fêmeas de um a doze dias, com percentuais de mortalidade maiores entre um e cinco dias (Tabela 1).

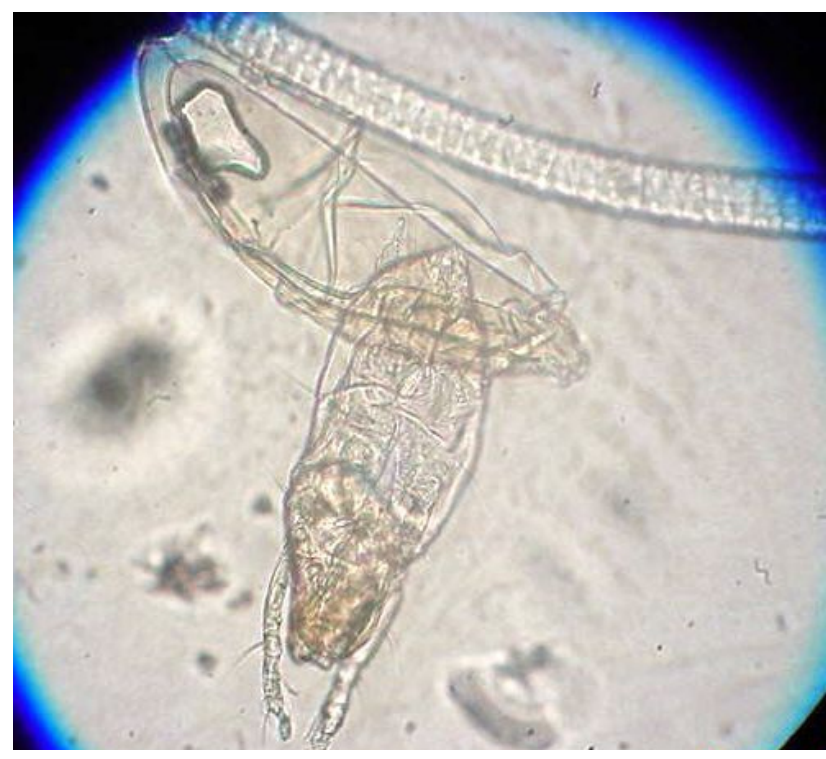

FIGURA 1. Larva do L. radovskyi presa à casca ovígera. (Objetiva $10 \mathrm{x}$ ) 
TABELA 1. Mortalidade dos diferentes estágios de L. radovskyi coletados de felinos naturalmente infestados, provenientes do campus da Universidade Federal Rural de Pernambuco, e mantidos em potes plásticos, sem o fornecimento de pelos, em temperatura ambiente (Fase 1)

\begin{tabular}{|c|c|c|c|c|c|c|c|c|c|c|c|c|}
\hline \multirow{3}{*}{ Dias } & \multicolumn{12}{|c|}{ Estágios } \\
\hline & \multicolumn{2}{|c|}{ Ovo/L } & \multicolumn{2}{|c|}{ Larva } & \multicolumn{2}{|c|}{ Ninfa } & \multicolumn{2}{|c|}{ Macho } & \multicolumn{2}{|c|}{ Fêmea } & \multicolumn{2}{|c|}{ Total } \\
\hline & $\mathrm{n}$ & $\%$ & $\mathrm{n}$ & $\%$ & $\mathrm{n}$ & $\%$ & $\mathrm{n}$ & $\%$ & $\mathrm{n}$ & $\%$ & $\mathrm{n}$ & $\%$ \\
\hline 1 & 23 & 100 & 1 & 20,0 & - & - & 10 & 17,2 & 10 & 17,2 & 44 & 20,5 \\
\hline 2 & - & - & 4 & 80,0 & - & - & 16 & 27,6 & 17 & 20,5 & 37 & 17,2 \\
\hline 3 & - & - & - & - & 6 & 13,0 & 21 & 36,2 & 15 & 18,1 & 42 & 19,5 \\
\hline 4 & - & - & - & - & 8 & 17,4 & 11 & 19,0 & 23 & 27,7 & 42 & 19,5 \\
\hline 5 & - & - & - & - & 15 & 32,6 & - & - & 10 & 12,0 & 25 & 11,6 \\
\hline 6 & - & - & - & - & 7 & 15,2 & - & - & 1 & 1,2 & 8 & 3,7 \\
\hline 7 & - & - & - & - & 4 & 8,7 & - & - & - & - & 4 & 1,9 \\
\hline 8 & - & - & - & - & 2 & 4,3 & - & - & 1 & 1,2 & 3 & 1,4 \\
\hline 9 & - & - & - & - & 2 & 4,3 & - & - & 3 & 3,6 & 5 & 2,3 \\
\hline 10 & - & - & - & - & 2 & 4,3 & - & - & 2 & 2,4 & 4 & 1,9 \\
\hline 12 & - & - & - & - & - & - & - & - & 1 & 1,2 & 1 & 0,5 \\
\hline Total & 23 & 100 & 5 & 100 & 46 & 100 & 58 & 100 & 83 & 100 & 215 & 100 \\
\hline
\end{tabular}

Ovo/L=lLarva eclodida do ovo coletado; larva= larva coletada; $\quad \mathrm{n}=$ frequência absoluta; $\%=$ frequência relativa. $\quad(-)$ Dado inexistente.

Na segunda fase, a taxa de eclosão em BOD foi de $100 \%$, sobrevivendo as larvas de um a dois dias. As larvas coletadas e submetidas à temperatura ambiente sobreviveram de um a dois dias, semelhante às larvas postas em BOD e às da fase 1 do experimento.

A sobrevivência das ninfas em temperatura ambiente foi de um a seis dias, e das colocadas em
BOD foi de um a cinco dias. As fêmeas sobreviveram de um a quatro e um a oito dias, respectivamente em temperatura ambiente e BOD. Para os machos, a sobrevivência tanto em ambiente quanto em BOD foi de um a quatro dias. As fêmeas submetidas à temperatura ambiente tiveram uma sobrevida menor quando comparadas com as da BOD (Tabelas 2 e 3 ).

TABELA 2. Mortalidade dos diferentes estágios de L. radovskyi coletados de felinos naturalmente infestados, provenientes do campus da Universidade Federal Rural de Pernambuco, e mantidos em tubos de Durhan, sem o fornecimento de pelos, em temperatura ambiente (Fase 2)

\begin{tabular}{|c|c|c|c|c|c|c|c|c|c|c|c|c|}
\hline \multirow[t]{3}{*}{ Dias } & \multicolumn{12}{|c|}{ Estágios } \\
\hline & \multicolumn{2}{|c|}{ Ovo/L } & \multicolumn{2}{|c|}{ Larva } & \multicolumn{2}{|c|}{ Ninfa } & \multicolumn{2}{|c|}{ Macho } & \multicolumn{2}{|c|}{ Fêmea } & \multicolumn{2}{|c|}{ Total } \\
\hline & $\mathrm{N}$ & $\%$ & $\mathrm{~N}$ & $\%$ & $\mathrm{~N}$ & $\%$ & $\mathrm{~N}$ & $\%$ & $\mathrm{n}$ & $\%$ & $\mathrm{n}$ & $\%$ \\
\hline 1 & - & - & 22 & 71,0 & 7 & 9,9 & 24 & 38,1 & 24 & 25,4 & 77 & 29,3 \\
\hline 2 & - & - & 9 & 29,0 & 31 & 43,7 & 18 & 28,6 & 33 & 33,7 & 91 & 34,6 \\
\hline 3 & - & - & - & - & 22 & 31,0 & 16 & 25,4 & 31 & 31,6 & 69 & 26,2 \\
\hline 4 & - & - & - & - & 9 & 12,7 & 5 & 7,9 & 10 & 10,2 & 24 & 9,1 \\
\hline 5 & - & - & - & - & 1 & 1,4 & - & - & - & - & 1 & 0,4 \\
\hline 6 & - & - & - & - & 1 & 1,4 & - & - & - & - & 1 & 0,4 \\
\hline Total & - & - & 31 & 100 & 71 & 100 & 63 & 100 & 98 & 100 & 263 & 100 \\
\hline
\end{tabular}

Ovo/ $\mathrm{L}=$ larva eclodida do ovo coletado; larva $=$ larva coletada $\quad \mathrm{n}=$ frequência absoluta ; \%= frequência relativa $\quad(-)$ dado inexistente. 
TABELA 3. Mortalidade dos diferentes estágios de L. radovskyi coletados de felinos naturalmente infestados, provenientes do campus da Universidade Federal Rural de Pernambuco, e mantidos em tubos de Durhan, sem o fornecimento de pelos, em BOD (Fase 2)

\begin{tabular}{|c|c|c|c|c|c|c|c|c|c|c|c|c|}
\hline \multirow[t]{3}{*}{ Dias } & \multicolumn{12}{|c|}{ Estágios } \\
\hline & \multicolumn{2}{|c|}{$\mathrm{Ovo} / \mathrm{L}$} & \multicolumn{2}{|c|}{ Larva } & \multicolumn{2}{|c|}{ Ninfa } & \multicolumn{2}{|c|}{ Macho } & \multicolumn{2}{|c|}{ Fêmea } & \multicolumn{2}{|c|}{ Total } \\
\hline & $\mathrm{n}$ & $\%$ & $\mathrm{n}$ & $\%$ & $\mathrm{n}$ & $\%$ & $\mathrm{n}$ & $\%$ & $\mathrm{n}$ & $\%$ & $\mathrm{n}$ & $\%$ \\
\hline 1 & 6 & 75,0 & 21 & 84,0 & 10 & 12,5 & 19 & 27,9 & 25 & 19,1 & 81 & 26,0 \\
\hline 2 & 2 & 25,0 & 4 & 16,0 & 34 & 42,5 & 23 & 33,8 & 32 & 24,4 & 95 & 30,4 \\
\hline 3 & - & - & - & - & 19 & 23,8 & 21 & 30,9 & 39 & 29,8 & 79 & 25,3 \\
\hline 4 & - & - & - & - & 12 & 15,0 & 5 & 7,4 & 17 & 13,0 & 34 & 10,9 \\
\hline 5 & - & - & - & - & 5 & 6,3 & - & - & 10 & 7,6 & 15 & 4,8 \\
\hline 6 & - & - & - & - & - & - & - & - & 1 & 0,8 & 1 & 0,3 \\
\hline 7 & - & - & - & - & - & - & - & - & 4 & 3,1 & 4 & 1,3 \\
\hline 8 & - & - & - & - & - & - & - & - & 3 & 2,3 & 3 & 1,0 \\
\hline Total & 8 & 100 & 25 & 100 & 80 & 100 & 68 & 100 & 131 & 100 & 312 & 100 \\
\hline
\end{tabular}

Ovo/L= larva eclodida do ovo coletado; larva= larva coletada; $\quad \mathrm{n}=$ frequência absoluta; $\%=$ frequência relativ ; (-) dado inexistente.

Dos resultados obtidos na terceira fase, a taxa de eclosão foi de $100 \%$, sobrevivendo as larvas de um a quatro dias, e as larvas coletadas de um a cinco dias. Para as ninfas, a sobrevida variou de um a 23 dias, e para os machos e fêmeas a sobrevida foi de um a 17 dias (Tabela 4). Constatou-se que a mortalidade foi mais tardia que as registradas na fase um (temperatura ambiente) e nas duas condições da fase dois (temperatura ambiente e BOD) do experimento.

TABELA 4. Mortalidade dos diferentes estágios de L. radovskyi coletados de felinos naturalmente infestados, provenientes do campus da Universidade Federal Rural de Pernambuco, e mantidos em tubos de Durhan, com fornecimento de pelos, em temperatura ambiente (Fase 3)

\begin{tabular}{|c|c|c|c|c|c|c|c|c|c|c|c|c|}
\hline \multirow[t]{3}{*}{ Dias } & \multicolumn{12}{|c|}{ Estágios } \\
\hline & \multicolumn{2}{|c|}{ Ovo/L } & \multicolumn{2}{|c|}{ Larva } & \multicolumn{2}{|c|}{ Ninfa } & \multicolumn{2}{|c|}{ Macho } & \multicolumn{2}{|c|}{ Fêmea } & \multicolumn{2}{|c|}{ Total } \\
\hline & $\mathrm{N}$ & $\%$ & $\mathrm{~N}$ & $\%$ & $\mathrm{~N}$ & $\%$ & $\mathrm{~N}$ & $\%$ & $\mathrm{~N}$ & $\%$ & $\mathrm{~N}$ & $\%$ \\
\hline 1 & 1 & 12,5 & 11 & 20,8 & 2 & 2,1 & 11 & 7,9 & 7 & 4,7 & 32 & 7,2 \\
\hline 2 & 2 & 25,0 & 9 & 17,0 & 10 & 10,6 & 24 & 17,3 & 26 & 17,3 & 71 & 16,0 \\
\hline 3 & 4 & 50,0 & 8 & 15,1 & 14 & 14,9 & 35 & 25,2 & 23 & 15,3 & 84 & 18,9 \\
\hline 4 & 1 & 12,5 & 8 & 15,1 & 9 & 9,6 & 18 & 12,9 & 16 & 10,7 & 52 & 11,7 \\
\hline 5 & - & - & 17 & 32,1 & 6 & 6,4 & 18 & 12,9 & 21 & 14,0 & 62 & 14,0 \\
\hline 6 & - & - & - & - & 16 & 17,0 & 6 & 4,3 & 18 & 12,0 & 40 & 9,0 \\
\hline 7 & - & - & - & - & 5 & 5,3 & 1 & 0,7 & 5 & 3,3 & 11 & 2,5 \\
\hline 8 & - & - & - & - & 7 & 7,4 & 3 & 2,2 & - & - & 10 & 2,3 \\
\hline 9 & - & - & - & - & 8 & 8,5 & 3 & 2,2 & 7 & 4,7 & 18 & 4,1 \\
\hline 10 & - & - & - & - & 7 & 7,4 & 4 & 2,9 & 10 & 6,7 & 21 & 4,7 \\
\hline 11 & - & - & - & - & 2 & 2,1 & 3 & 2,2 & 6 & 4,0 & 11 & 2,5 \\
\hline 12 & - & - & - & - & 2 & 2,1 & 2 & 1,4 & - & - & 4 & 0,9 \\
\hline 13 & - & - & - & - & 2 & 2,1 & 4 & 2,9 & 2 & 1,3 & 8 & 1,8 \\
\hline 14 & - & - & - & - & 1 & 1,1 & - & - & - & - & 1 & 0,2 \\
\hline 15 & - & - & - & - & 1 & 1,1 & 5 & 3,6 & 4 & 2,7 & 10 & 2,3 \\
\hline 16 & - & - & - & - & - & - & - & - & 2 & 1,3 & 2 & 0,5 \\
\hline 17 & - & - & - & - & 1 & 1,1 & 2 & 1,4 & 3 & 2,0 & 6 & 1,4 \\
\hline 23 & - & - & - & - & 1 & 1,1 & - & - & - & - & 1 & 0,2 \\
\hline Total & 8 & 100 & 53 & 100 & 94 & 100 & 139 & 100 & 150 & 100 & 444 & 100 \\
\hline
\end{tabular}

Ovo/L= larva eclodida do ovo coletado; larva=larva coletada; $\mathrm{n}=$ frequência absoluta $\%=$ frequência relativa $\quad(-)$ dado inexistente. 
Através do teste de Tukey, visando a comparação pareada entre as médias dos períodos de sobrevivência dos estágios, para a fase 1 , observou-se que não houve diferença significativa entre a sobrevivência da larva eclodida no laboratório e da larva coletada do animal parasitado. No entanto, ambas diferiram dos outros estágios, exceto em relação às larvas coletadas e machos. As ninfas tiveram média de sobrevivência significativamente maior que os demais estágios e as fêmeas apresentaram sobrevivência estatisticamente maior que os machos (Tabela 5).

Houve diferença significativa entre as médias do período de sobrevivência das larvas coletadas em relação aos demais estágios na fase 2 , não havendo diferença entre ninfas e fêmeas, e machos e fêmeas. Em BOD, houve diferença significativa entre a sobrevivência média da larva eclodida e da larva coletada, quando comparada aos demais estágios, e entre machos e fêmeas, com sobrevida maior para as fêmeas.
Comparando-se os dois ambientes experimentais, apenas para as fêmeas houve diferença significativa entre as médias, sendo maior a sobrevivência para os espécimes mantidos em BOD (Tabela 5).

$\mathrm{Na}$ fase 3, observou-se diferença significativa entre as médias das larvas eclodidas e as larvas coletadas, porém diferiram dos demais estágios, semelhante ao que ocorreu nas fases 1 e 2 do experimento. As ninfas apresentaram período médio de sobrevivência significativamente maior quando comparado aos dos outros estágios, exceto ao das fêmeas. Os resultados observados nesta fase foram semelhantes aos achados nas outras fases do experimento, porém com a particularidade de que todos os estágios tiveram uma sobrevida média bem superior às médias das fases anteriores. A média de sobrevivência dos machos não diferiu estatisticamente da das fêmeas, porém diferiu dos estágios restantes (Tabela 5).

TABELA 5. Média e desvio padrão dos períodos de sobrevivência dos diferentes estágios de L. radovskyi coletados de felinos naturalmente infestados, provenientes do campus da Universidade Federal Rural de Pernambuco, mantidos em temperatura ambiente e em $\mathrm{BOD}$, segundo as fases

\begin{tabular}{|c|c|c|c|c|}
\hline & \multirow[t]{2}{*}{ Estágio } & \multicolumn{2}{|c|}{ dias } & \multirow[t]{2}{*}{ Valor de $p$} \\
\hline & & Temperatura ambiente & B.O.D. & \\
\hline \multirow[t]{5}{*}{ Fase 1} & Ovo/L & $1,00 \pm 0,00^{(\mathrm{A})}$ & - & - \\
\hline & Larva & $1,80 \pm 0,45^{(\mathrm{AD})}$ & - & - \\
\hline & Ninfa & $5,41 \pm 1,81^{(\mathrm{B})}$ & - & - \\
\hline & Macho & $2,57 \pm 0,99^{(\mathrm{CD})}$ & - & - \\
\hline & Fêmea & $3,66 \pm 2,22^{(\mathrm{E})}$ & - & - \\
\hline Valor de $\mathrm{p}$ & & $\mathrm{P}^{(3)}<0,001^{*}$ & & \\
\hline \multirow[t]{5}{*}{ Fase 2} & Ovo/L & & $1,70 \pm 0,73^{(\mathrm{A})}$ & $* *$ \\
\hline & Larva & $1,29 \pm 0,46^{(\mathrm{A})}$ & $1,16 \pm 0,37^{(\mathrm{A})}$ & $\mathrm{p}^{(1)}=0,248$ \\
\hline & Ninfa & $2,56 \pm 0,98^{(\mathrm{B})}$ & $2,60 \pm 1,09^{(\mathrm{BD})}$ & $\mathrm{p}^{(2)}=0,829$ \\
\hline & Macho & $2,03 \pm 0,98^{(\mathrm{CD})}$ & $2,18 \pm 0,93^{(\mathrm{B})}$ & $\mathrm{p}^{(2)}=0,388$ \\
\hline & Fêmea & $2,28 \pm 0,95^{(\mathrm{BD})}$ & $2,92 \pm 1,61^{(\mathrm{CD})}$ & $\mathrm{p}^{(1)}<0,001^{*}$ \\
\hline Valor de $\mathrm{p}_{3}$ & & $\mathrm{p}^{(3)}<0,001^{*}$ & $\mathrm{p}^{(3)}<0,001^{*}$ & \\
\hline \multirow[t]{5}{*}{ Fase 3} & Ovo/L & $2,63 \pm 0,92^{(\mathrm{A})}$ & & - \\
\hline & Larva & $2,31 \pm 1,56^{(\mathrm{A})}$ & & - \\
\hline & Ninfa & $6,38 \pm 3,82^{(\mathrm{B})}$ & & - \\
\hline & Macho & $4,88 \pm 3,79^{(\mathrm{C})}$ & & - \\
\hline & Fêmea & $5,59 \pm 3,86^{(\mathrm{BC})}$ & & - \\
\hline Valor de $\mathrm{p}$ & & $\mathrm{p}^{(3)}<0,001^{*}$ & & \\
\hline
\end{tabular}

Ovo/L= larva eclodida do ovo coletado; (-) dado inexistente.

(*) Diferença significativa a $5,0 \%$.

(**) Não foi determinado, em virtude da ocorrência de frequência nula ou muito baixa.

$\mathrm{p}_{1}=$ Relativo à comparação entre as condições experimentais através do teste t-Student com variâncias desiguais.

$\mathrm{p}_{2}=$ Relativo à comparação entre as condições experimentais através do teste t-Student com variâncias iguais.

$\mathrm{P}_{3}=$ Relativo à comparação entre os estágios para cada condição experimental através do teste $\mathrm{F}$ (ANOVA).

Obs.: Se nenhuma letra entre parênteses for comum, existe diferença significativa entre os estágios correspondentes através das comparações de Tukey. 
Embora não existam registros sobre tempos de sobrevivência de L. radovskyi, LIGNON et al. (1994), trabalhando com Sarcoptes scabiei, observaram sobrevivência maior fora do hospedeiro, de ninfas e fềmeas, as quais apresentaram média de seis dias a $25 \pm 1^{\circ} \mathrm{C}$ e $75 \%$ de umidade relativa e sete dias a $20 \pm 1{ }^{\circ} \mathrm{C}$ e $75 \%$, quando comparadas aos machos, que morreram todos no decorrer de 1,74 dias a $25 \pm 1{ }^{\circ} \mathrm{C}$ e $75 \%$ e quatro dias a $20 \pm 1{ }^{\circ} \mathrm{C}$ e $75 \%$.

Na Tabela 6, consta a comparação do tempo de sobrevivência das fases 1 e 3, que transcorreram no am- biente, segundo o estágio. Comprovaram-se diferenças significativas entre os dois experimentos para cada um dos estágios analisados, observando-se que as médias da fase 3 do experimento foram correspondentemente mais elevadas do que as do experimento 1. A adição do pelo felino como substrato pode ter contribuído para a diferença obtida. Deve-se, porém, levar também em consideração as diferenças de temperatura e umidade relativa observadas entre as épocas de realização dos experimentos.

TABELA 6. Média de sobrevivência dos diferentes estágios de L. radovskyi coletados de felinos naturalmente infestados, provenientes do campus da Universidade Federal Rural de Pernambuco, e mantidos em temperatura ambiente, sem pelos (Fase 1) e com pelos (Fase 3), segundo o estágio e a fase

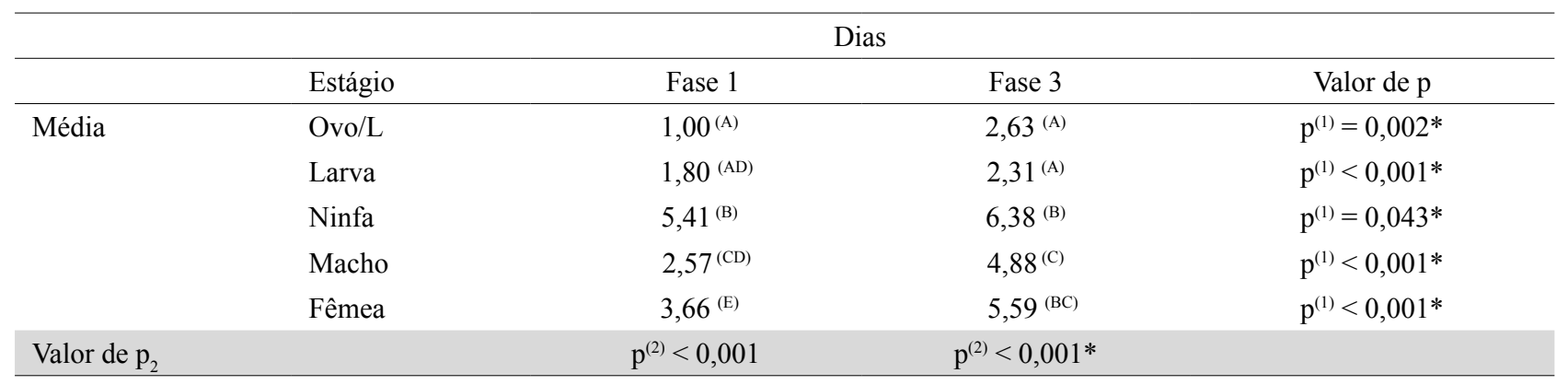

Ovo/L= Larva eclodida do ovo coletado.

(*) Diferença significativa a $5,0 \%$.

$\mathrm{p}_{1}=$ Relativo à comparação para os estágios das fases experimentais 1 e 3 através do teste t-Student com variâncias desiguais.

$\mathrm{p}_{2}=$ Relativo à comparação entre as condições experimentais através do teste t-Student com variâncias iguais.

Obs.: Se nenhuma letra entre parênteses for comum, existe diferença significativa entre os estágios correspondentes através das comparações de Tukey.

Quanto aos ovos coletados, pôde-se observar que, inicialmente, apresentavam coloração vítrea brilhante, a qual, com o passar do tempo, modificou-se para opaca e, posteriormente, escurecida, tendendo à cor marrom, até a eclosão das larvas.

Não se observou a realização de postura por parte das fêmeas em nenhuma das três fases do experimento, seja das fêmeas mantidas individualmente ou das postas junto aos machos. Todavia observou-se atitude possivelmente de cortejo por parte dos machos de todos os seis casais, os quais se posicionavam sobre o dorso das fêmeas (Figura 2). Dentre os casais, a sobrevivência das fêmeas foi de um a oito dias, sendo mais tardia que a dos machos (um a cinco dias).

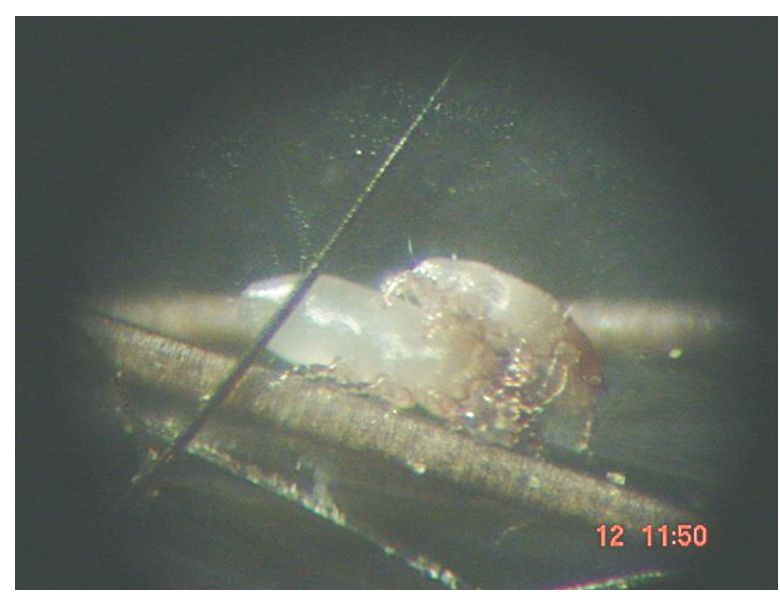

FIGURA 2. Posicionamento do macho sobre a fêmea de L. radovskyi. (Microscópio estereoscópico) 


\section{CONCLUSÃO}

Os estágios de ovo, larva, ninfas, machos e fêmeas do L. radovskyi sobrevivem fora do hospedeiro. A ausência ou presença de pelo felino parece ser fator importante na duração dos períodos de sobrevivência destes estágios.

\section{REFERÊNCIAS}

ALTMAN, D. G. Practical statistics for medical research. London, Great Britain: Chapman \& Hall/CRC Texts in Statistical Science, 1991. $611 \mathrm{p}$.

CRAIG, T. M.; DUBUISSON, L. M.; DUBUISSON, R. K. Lynxacarus radovskyi infestation in a cat. Journal American Veterinary Medical Association, v. 202, n. 4, p. 613-614, 1993.

CROCE, J.; BAGGIO, D. Ácaros contaminantes de ambientes e causadores de doenças alérgicas no homem. Revista Brasileira de Alergia e Imunopatologia, v. 16, p. 219-220, 1993.

FACCINI, J. L. H.; COUTINHO, V. Ocorrência de Lynxacarus radovskyi (Acari: Listrophoridae) em gatos domésticos no Brasil. Arquivo da Universidade Federal do Rio de Janeiro, UFRRJ, v. 9, n. 1-2, p. 91-93, 1986.
FLECHTMANN, C. H. W. Elementos de acarologia. São Paulo: Nobel, 1975. 344 p.

FOLEY, R. H. An epizootic of rare fur mite in an island's cat population. Feline Practice, v. 19, n. 3, p. 17-19, 1991.

GUIMARÃES, J. H.; TUCCI, E. C.; BARROS-BATTESTI, D. M. Ectoparasitos de importância veterinária. São Paulo: FAPESP, 2001.

LIGNON, G. B.; FACCINI, J. L. H.; BARBOSA, C. G. Sobrevivência de adultos, ninfas e larvas de Sarcoptes scabiei (L., 1758) fora dos hospedeiros e em condições controladas. Revista Brasileira de Parasitologia Veterinária, v. 3, n.1, p. 57-60, 1994.

PEREIRA M. C. The Cat fur mite (Lynxacarus radovskyi) in Brazil. Feline Practice Parasitology, v. 24, n. 5, p. 24-26, 1996.

SCOTT, D. W. Feline dermatology: a monograph. Journal American Animal Hospital Association, v. 16, p. 331, 1980.

SLOSS, M. W.; ZAJAC, A. N.; KEMP, R. L. Parasitologia clínica veterinária. 6. ed. São Paulo: Manole, 1999. 198 p.

TENORIO, J. M. A New Species of Lynxacarus (Acarina: Astigmata: Listrophoridae) from Felis catus in the Hawaiian Islands. Journal of Medical Entomology, v. 11, p. 599-604, 1974.

ZAR, J.H. Biostatistical analysis. 4. ed. New Jersey, USA: Prentice Hall, 1999. $929 \mathrm{p}$.

Protocolado em: 9 fev. 2009. Aceito em: 27 jan. 2010. 